\title{
Metal nanoring and tube formation on carbon nanotubes
}

\author{
V. M. K. Bagci, ${ }^{1}$ O. Gülseren, ${ }^{2,3}$ T. Yildirim, ${ }^{2}$ Z. Gedik, ${ }^{1}$ and S. Ciraci ${ }^{1}$ \\ ${ }^{1}$ Department of Physics, Bilkent University, Ankara 06533, Turkey \\ ${ }^{2}$ NIST Center for Neutron Research, National Institute of Standards and Technology, Gaithersburg, Maryland 20899 \\ ${ }^{3}$ Department of Materials Science and Engineering, University of Pennsylvania, Philadelphia, Pennsylvania 19104
}

(Received 18 March 2002; revised manuscript received 23 May 2002; published 18 July 2002)

\begin{abstract}
The structural and electronic properties of aluminum-covered single-wall carbon nanotubes (SWNT's) are studied from first principles for a large number of coverages. Aluminum-aluminum interaction, that is stronger than aluminum-tube interaction, prevents uniform metal coverage, and hence gives rise to the clustering. However, a stable aluminum ring and aluminum nanotube with well defined patterns can also form around the semiconducting SWNT's and lead to metallization. The persistent current in the Al nanoring is discussed to show that a high magnetic field can be induced at the center of SWNT.
\end{abstract}

DOI: 10.1103/PhysRevB.66.045409

PACS number(s): 73.22. $-\mathrm{f}, 61.48 .+\mathrm{c}, 71.30 .+\mathrm{h}, 73.20 . \mathrm{Hb}$

\section{INTRODUCTION}

Stable metal wires, having diameters in the range of nanometers, are very important for nanoelectronics and other nanodevice applications. The metal nanowires ${ }^{1,2}$ and monoatomic chains ${ }^{3,4}$ produced so far have played a crucial role in understanding quantum transport and exotic atomic structure. ${ }^{5-8}$ Earlier, those wires were neither reproducible nor controllable to offer any relevant technological application. Recently, it was shown that such nanowires can be produced by depositing metal atoms on carbon nanotube templates. ${ }^{9,10}$ Because of its curvature, the surface of a single-wall carbon nanotube (SWNT) is chemically more reactive than graphite. Therefore, stable bonding can occur between the SWNT and metal adatom. ${ }^{11-13}$ Recently, Mo-Ge superconducting nanowires were fabricated using sputter deposition on carbon nanotubes. ${ }^{14} \mathrm{~A}$ continuous titanium coating of varying thicknesses, and quasicontinuous coatings of $\mathrm{Ni}$ and $\mathrm{Pd}$, were obtained by using electron-beam evaporation techniques, ${ }^{9,10}$ whereas $\mathrm{Au}, \mathrm{Al}, \mathrm{Fe}$, and $\mathrm{Pd}$ were able to form only discrete particles or clusters rather than a continuous coating of the SWNT. Nevertheless, the coating of virtually any metal on SWNT can be mediated by first depositing titanium as a buffer layer., ${ }^{9,10,15}$

SWNT's seem to be ideal templates for synthesizing a variety of stable nanowires with different diameters, thicknesses, and lengths of elemental metals. It is therefore important to have a good understanding of metal-SWNT interactions and the mechanism of metal coverage. In this paper, we address this issue from first principles by studying structural and electronic properties of $\mathrm{Al}$ adsorption, starting from single-atom adsorption to monolayer coverage. We find that the $\mathrm{Al}-\mathrm{Al}$ interaction is relatively stronger than the Al-SWNT interaction, yielding Al-cluster formation rather than a uniform coating over the SWNT for most cases. However, we discover that a stable $\mathrm{Al}$ nanoring and also an $\mathrm{Al}$ tube can form at well-defined and ordered positions over the $(8,0)$ SWNT. Furthermore, we estimate that the current through the $\mathrm{Al}$ nanorings can produce large magnetic fields at the tip of a nanotube. We hope that these findings will shed light on the usage of nanotubes as a template to grow metal nanowires with many interesting properties.

\section{METHOD}

First-principles total-energy and electronic structure calculations were performed using the pseudopotential planewave method ${ }^{16}$ within the generalized gradient approximation. ${ }^{17} \mathrm{~A}$ tetragonal supercell was used with lattice constants $a_{s c}=b_{s c} \sim 22 \AA$ and $c_{s c}$. The latter is taken to be equal to the one-dimensional (1D) lattice parameter $c$ of the tube. To minimize the adsorbate-adsorbate interaction, some calculations are performed in longer supercells by taking $c_{s c}=2 c$. We use ultrasoft pseudopotentials ${ }^{18}$ for carbon and aluminum atoms, and plane waves up to an energy cutoff of $310 \mathrm{eV}$. Brillouin-zone integrations are performed with 12-6 special $\mathbf{k}$ points. All atomic positions of adsorbate and nanotube as well as $c$ are fully optimized.

\section{RESULTS AND DISCUSSION}

We first explored the possible adsorption sites for an individual $\mathrm{Al}$ atom on a $(8,0)$ nanotube; namely $H$ sites which are above the center of hexagons, $Z$ and $B$ sites which are above the zigzag and axial C-C bonds, respectively, and finally $T$ sites which are on top of the carbon atoms. The binding energy is obtained from the expression

$$
E_{b}=E_{T}[\mathrm{SWNT}]+E_{T}[\mathrm{Al}]-E_{T}[\mathrm{Al}+\mathrm{SWNT}]
$$

in terms of the total energies of the fully optimized bare nanotube $\left(E_{T}[\mathrm{SWNT}]\right)$, the atomic $\mathrm{Al}\left(E_{T}[\mathrm{Al}]\right)$, and the $\mathrm{Al}$-adsorbed nanotube $\left(E_{T}[\mathrm{Al}+\mathrm{SWNT}]\right)$. All total-energy calculations are carried out in the same supercell with $c_{s c}$ $=2 c$. According to the above definition stable structures have positive binding energies. We find that the bindings at $T$ sites are unstable; the adatoms move to the $H$ sites upon relaxation. The bindings at $H, Z$, and $B$ sites are found to be stable with C-Al distances of $2.28,2.30$, and $2.25 \AA$, respectively. The corresponding binding energies are 1.70, 1.54, and $1.60 \mathrm{eV}$, respectively. ${ }^{19}$ According to Mulliken analysis, $\sim 0.7 e$ is transferred to the nanotube upon absorption of a single $\mathrm{Al}$ atom, and partially occupied electronic states occur in the band gap. While the binding energy of $\mathrm{Al}$ is negligible on the graphite surface, the curvature of the $(8,0)$ tube provides a significant binding interaction. ${ }^{11,13,20}$ 
(a)

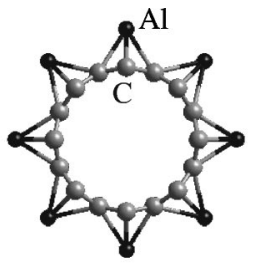

(c)

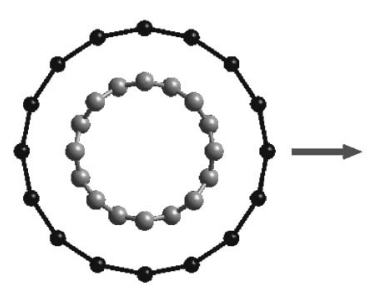

(b)

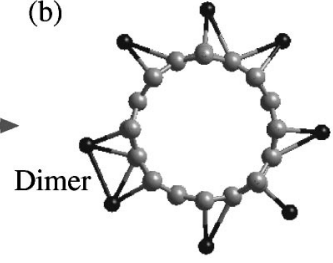

(d)

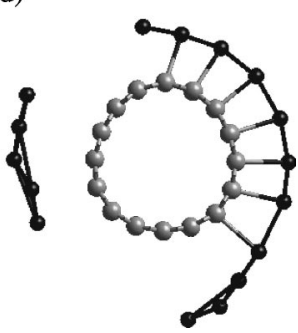

FIG. 1. (a) Initial structure of the Al ring where the adatoms were placed at $\mathrm{H}$ sites on the circumference of the tube. (b) Dimerization upon relaxation of $\mathrm{Al}$ atoms starting from the structure shown in (a). (c) Initial structure of the uniform coverage of a nanotube where all $\mathrm{H}$ sites are occupied by $\mathrm{Al}$ atoms. (d) The nucleation of isolated $\mathrm{Al}$ clusters from the initial structure shown in (c). Both relaxed structures shown in (b) and (d) are not final equilibrium structures, but they are intermediate configurations toward a $3 \mathrm{D}$ cluster formation.

After having discussed the adsorption of a single $\mathrm{Al}$ atom on a $(8,0)$ nanotube, we next consider the adsorption of several $\mathrm{Al}$ atoms where $\mathrm{Al}-\mathrm{Al}$ interactions play an important role. Since the binding energy at the $H$ sites is the largest, we first consider a coverage where $\mathrm{Al}$ atoms are placed at $H$ sites. We start with a quarter-coverage case (i.e., $\Theta=0.25$ ) by placing eight $\mathrm{Al}$ atom at the $H$ sites around the circumference, forming a ring in the double unit cell of the $(8,0)$ nanotube. According to this initial structure the $\mathrm{Al}-\mathrm{Al}$ and C-Al distances are 3.7 and $2.4 \AA$, respectively. Once this system (consisting of $64 \mathrm{C}$ and eight $\mathrm{Al}$ atoms) is relaxed, we find that some $\mathrm{Al}$ atoms move away from the nanotube surface towards their neighbors and eventually form a dimer, as shown in Figs. 1(a)-1(b). We attribute this dimerization to the $\mathrm{Al}-\mathrm{Al}$ interaction, which is stronger than the Al-nanotube interactions.

A uniform half-coverage (i.e., $\Theta=0.5$ ), where initially all $H$ sites are occupied by $\mathrm{Al}$ atoms (i.e., $32 \mathrm{C}$ and $16 \mathrm{Al}$ atoms) also exhibits instability. Upon relaxation of this system, $\mathrm{Al}$ atoms tend to reduce the $\mathrm{Al}-\mathrm{Al}$ distance from 3.0 to 2.5-2.8 $\AA$, and at the same time they rise above the surface of the tube. At the end small and isolated clusters form on the nanotube. Some of the adatoms become completely disconnected from the surface to initiate a 3D island growth, since the latter is energetically favorable with a binding energy $E_{b}$ $>3 \mathrm{eV}$. This situation, as illustrated in Figs. 1(c) $-1(\mathrm{~d})$, is in good agreement with the experimental observations., ${ }^{9}$

Above we showed that a uniform Al coverage on the $H$ sites did not yield a stable structure due to strong Al-Al interactions. However, if the $\mathrm{Al}$ atoms are placed at the $T$ sites (i.e., on top of the carbon atoms), the $\mathrm{Al}-\mathrm{Al}$ distance no longer has to be large. Therefore, one can optimize the Al-Al and Al-nanotube interactions simultaneously. Below we

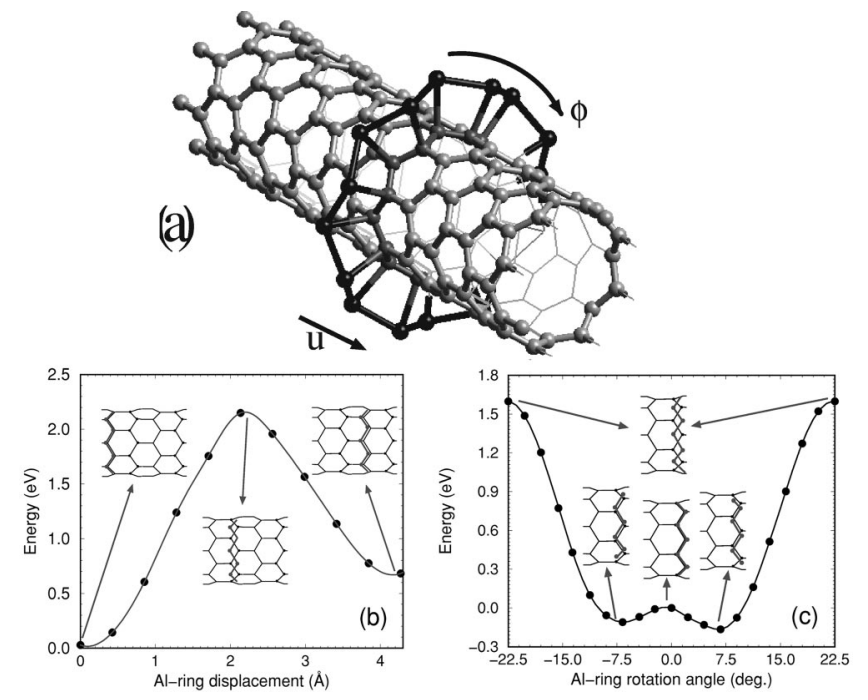

FIG. 2. (a) A view of the optimized structure of the Al zigzag nanoring formed on a $(8,0)$ SWNT. (b) Variation of the (relative) energy with the rigid displacement $u$ of the $\mathrm{Al}$ ring along the tube axis. The starting point is the optimized structure. The insets show schematic views of a nanoring (thick gray lines) and a carbon nanotube for three particular configurations. (c) Variation of the energy with rigid rotation $\phi$ of the $\mathrm{Al}$ ring around the nanotube. The right minimum at $\phi=7^{\circ}$ corresponds to the optimized structure; $\phi=0^{\circ}$ is the ideal configuration, with $\mathrm{Al}$ atoms aligned perfectly on top of the carbon atoms.

demonstrate this situation for two special coverage cases, namely, a zigzag $\mathrm{Al}$ nanoring and an $\mathrm{Al}$ nanotube around the $(8,0)$ SWNT.

We first discuss the zigzag $\mathrm{Al}$ nanoring coverage, which is obtained by placing $\mathrm{Al}$ atoms on top of carbon atoms ( $T$ site), forming a zigzag ring [see Fig 2(a)]. This structure includes $64 \mathrm{C}$ and $16 \mathrm{Al}$ atoms in the double unit cell. In this initial configuration the $\mathrm{Al}-\mathrm{Al}$ distance is $2.33 \AA$, and the angle of $\mathrm{Al}-\mathrm{Al}-\mathrm{Al}$ bond is $\sim 137^{\circ}$. After structure optimization, the Al-Al bond length is increased to $2.56 \AA$, and the $\mathrm{Al}-\mathrm{Al}-\mathrm{Al}$ bond angle is decreased to $124^{\circ}$, yielding the radius of the nanoring to be $5.9 \AA$. A side view of the optimized structure of the Al nanoring wrapping the $(8,0)$ SWNT is illustrated in Fig. 2(a). The binding energy of the Al nanoring is calculated to be $0.85 \mathrm{eV}$. The stability of the Al nanoring around the nanotube can be understood from the stable structures of planar Al monoatomic chains. Recent studies of the low-dimensional structures of metals revealed several stable atomic structures in one dimension. ${ }^{6,8,21-25}$ The first-principles calculations predicted linear chain, planar zigzag, triangular, ladder and nonplanar dumbbell and pentagonal structures as stable structures for Al wires. ${ }^{6,21,25}$ More interestingly, it was found that by going from a bulk structure to a chain structure the character of bonding in $\mathrm{Al}$ wires changes and acquires directionality. ${ }^{25}$ Among a number of these 1D stable structures of Al predicted by first-principles calculations $^{25}$ was a planar zigzag monoatomic chain of $\mathrm{Al}$ with a bond angle of $139^{\circ}$ and a bond distance of $2.53 \AA$. This zigzag structure is only a local minimum on the BornOppenheimer surface, and hence its binding energy (1.92 $\mathrm{eV}$ ) is intermediate between the binding energies of bulk and 
linear structures. An energy barrier of $\sim 0.1 \mathrm{eV}$ prevents the transition from the planar zigzag structure to other relatively more stable 1D structures. ${ }^{25}$ The final optimized structure of the zigzag $\mathrm{Al}$ nanoring around the $(8,0)$ nanotube has structural parameters similar to this planar zigzag structure, except that it is rolled on a cylinder. The SWNT initially serves as a template in the formation of the ring structure, and also increases the stability of the ring by preventing transitions to other relatively more stable structures. Therefore, the Al nanoring around the SWNT is expected to be stable at room temperature. Interestingly, the nanoring is also stable by itself, since the positions of $\mathrm{Al}$ atoms do not change significantly upon discarding the underlying carbon nanotube.

We further analyzed the SWNT-Al nanoring interaction by studying the effect of the rigid displacement $(u)$ and rotation $(\phi)$ of the nanoring around the tube axis. The variation of the energy as a function of displacement, $E(u)$ $=E_{T}^{u}[\mathrm{Al}+\mathrm{SWNT}, u]-E_{T}[\mathrm{Al}+\mathrm{SWNT}]$ is shown in Fig. 2(b). Here $E_{T}^{u}[\mathrm{Al}+\mathrm{SWNT}, u]$ is the total energy of an unrelaxed tube-ring system with the ring displaced by $u$. The highest energy configuration (energetically least favorable) corresponds to the situation where $\mathrm{Al}$ atoms are close to the $H$ sites. For $u=c, E$ is $0.7 \mathrm{eV}$ higher than the initial energy with optimized structure, $E(u=0)$, since no structure optimization was done at $u=c$. The rigid rotation of the ring in the interval $-22.5^{\circ} \leqslant \phi \leqslant 22.5^{\circ}$ is also shown in Fig. 2(c). The highest energy configuration again corresponds to the case where $\mathrm{Al}$ atoms are close to the $H$ sites [see Fig. 2(b)]. When the ring is aligned perfectly on the top of carbon atoms at $\phi=0^{\circ}$, we obtain a saddle point. Rotations by $\phi \sim \pm 7^{\circ}$ from the ideal configuration $\left(\phi=0^{\circ}\right)$ result in two stable configuration with a double minimum potential separated by a barrier of $0.2 \mathrm{eV}$. The right minimum at $\phi=7^{\circ}$ corresponds to the starting optimized structure.

The electronic properties of the zigzag Al nanoring system described above [Fig. 2(a)] is also quite interesting, and may lead to important applications. The electronic energy bands of the Al metal ring (without SWNT's) are derived from the dispersive bands of the flat zigzag Al chain. ${ }^{25}$ When the flat zigzag $\mathrm{Al}$ chain is rolled into a ring, its bands are zone folded at the $\Gamma$ point, and they appear as a number of discrete energy levels as shown in Fig. 3. For the case of the Al nanoring wrapping the nanotube, shown in Fig. 2(a), these states are mixed with the states of the nanotube and give rise to the bands and density of states shown in Fig. 3. As a result of $\mathrm{Al}$ nanoring and $(8,0)$ nanotube interaction, the combined system is a metal. The small dispersion of the bands associated with the nanoring is due to the small interaction between the nanorings in the supercell. The dispersion of these bands is reduced with the increasing ring-ring distance, and eventually becomes localized states of a single ring which dopes the empty conduction bands of the $(8,0)$ SWNT. According to Mulliken analysis 0.15 electrons are transferred from each $\mathrm{Al}$ atom to the SWNT. Most importantly, the $\mathrm{Al}$ ring is a conductor that incorporates two channels with an ideal ballistic quantum conductance of $4 e^{2} / h$.

The small radius of the metallic nanoring wrapping the carbon nanotube may lead to interesting electromagnetic



FIG. 3. The energy bands along the nanotube axis of the Al nanoring formed on a $(8,0)$ SWNT (middle panel). The total density of states is shown in the left panel. The right panel shows the energy levels of the bare $\mathrm{Al}$ nanoring. The zero energy is taken at the Fermi level.

properties. The magnetic field $B$ at the center of the ring can be expressed in terms of the quantized angular momentum $L_{z}$ of the electrons in the direction paralel to the tube axis,

$$
B=\frac{\mu_{0} e L_{z}}{4 \pi m r^{3}},
$$

where $r$ is the radius of the nanoring. Taking the lowest possible value for $L_{z}$ and $r=5.9 \AA$, we estimate $B$ to be of the order of 100 gauss. The current in the metal ring that can induce such a high magnetic field is comparable to the current attained in the suspended, monoatomic gold chains. ${ }^{4}$ Relatively higher magnetic fields at the order of $1 \mathrm{~T}$ can be induced by higher current passing through a thick titanium based metal coating around the SWNT, or by increasing the number of turns and hence by forming a nanocoil. Miyamoto et $a l .{ }^{26}$ examined the chiral conductivity in bare $\mathrm{BC}_{2} \mathrm{~N}$ nanotubes. They estimated that magnetic field of a few tenths of 1 $\mathrm{T}$ can be induced at the center of the tube by assuming a relaxation time of carriers $\sim 50$ times larger than that in $\mathrm{Cu}$, and a homogeneous chiral current density confined to the tubule wall.

Persistent currents in the nanoring can also start by a sudden application of an external magnetic field. In this way it is possible to use a nanotube, with a ring at its end, as a local magnetic probe at a nanoscale. A superconducting ring may also be used for Schrödinger cat experiments, where one deals with superposition of macroscopic quantum states. ${ }^{27}$ The two supercurrent quantum states (clockwise and counterclockwise flow) sit in two separate quantum wells. It has been observed that a weak microwave, which does not break Cooper pairs, can cause quantum tunneling between these two macroscopic states. In this kind of experiment the main problem is to isolate the superconducting quantum interference device from the outside (nonquantum) environment, and that is why isolated carbon nanotubes can be very useful. 
(a)

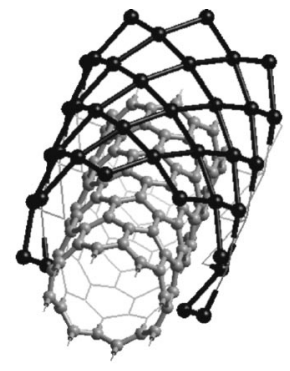

(b)
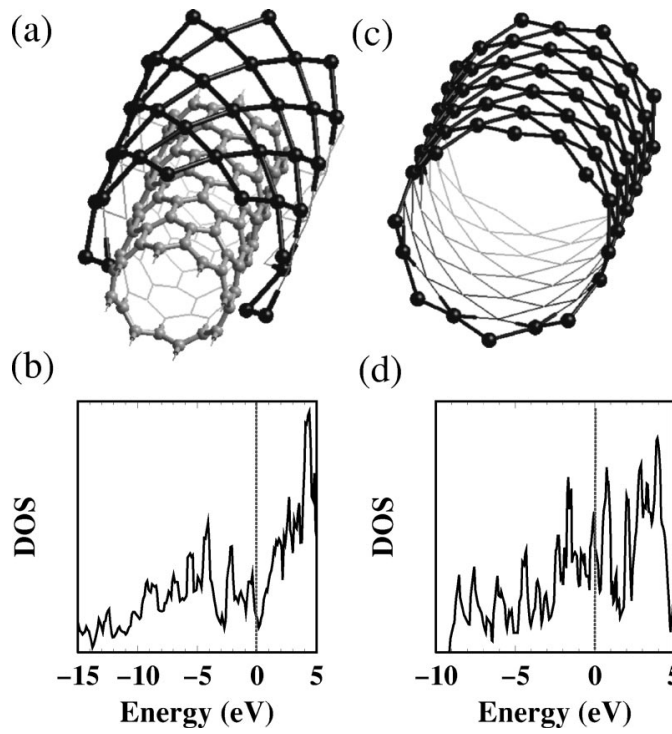

(d)

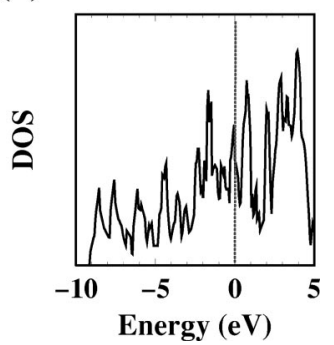

FIG. 4. (a) Aluminum nanowire (dark) around a SWNT (gray). (b) Total density of state for an Al nanowire + SWNT structure. (c) Structure of an Al nanowire alone, which is also stable with a smaller radius. (d) Total DOS for the Al nanowire shown in (c). The zero energy is taken at $E_{F}$.

Finally we discuss another stable uniform coverage which is obtained from $16 \mathrm{Al}$ atoms placed at alternating $T$ sites in one unit cell. Upon relaxing this structure, $\mathrm{Al}$ atoms move toward the bridge sites so that the nearest-neighbor distance is $2.7 \AA$ (which is close to that of the bulk Al nearest neighbor distance, $d_{o}=2.8 \AA$ ) and the Al-tube distance is $2.4 \AA$. The final, stable structure can be viewed as a tubular $\mathrm{Al}$ (i.e., an $\mathrm{Al}$ nanotube), the structure of which is matched to the SWNT with a significant tube-Al interaction (Fig. 4). This structure is a metal with a finite density of states at $E_{F}$ [Fig. 4(c)]. To check whether the $\mathrm{Al}$ nanotube is stable and can maintain its structure without the underlying SWNT, we optimized the same structure without a SWNT. Apart from decreasing the radius of the metal nanotube, the geometric structure is preserved. This indicates that the tubular $\mathrm{Al}$ is stable by itself, and the interaction between the SWNT and

the $\mathrm{Al}$ nanowire is not against the $\mathrm{Al}-\mathrm{Al}$ stability. The density of states of the bare Al nanowire without a SWNT indicates that the system is also a metal [Fig. 4(d)].

\section{CONCLUSIONS}

In conclusion, we show that normally $\mathrm{Al}$ atoms do not form a uniform metal coverage, but rather tend to nucleate isolated clusters on the surface of a SWNT, in agreement with experiments. ${ }^{9,10}$ This is due to the fact that the Al-Al interaction is stronger than that of the Al SWNT, and for most of the decorations of $\mathrm{Al}$ atoms on SWNT these two interactions compete. However, we found two special Al coverages, namely, a SWNT wrapped by a zigzag Al ring and a SWNT covered by a uniform and concentric Al nanotube. In these systems the $\mathrm{Al}-\mathrm{Al}$ and $\mathrm{Al}$-nanotube interactions are not frustrated, which is the main reason for the stability. In both cases we find significant charge transfer from $\mathrm{Al}$ to the SWNT, leading to a metalization of the semiconducting tubes. An Al nanoring around a SWNT is of particular interest because the states of the ring near the Fermi level can carry ballistic current around the ring. We show that this can give rise to persistent current and/or high magnetic fields along the axis of the tube. Clearly this is a very promising effect for many nanodevice applications, and provides tools for experiments on fundamental aspects of quantum mechanics. In spite of the fact that $\mathrm{Al}$ is highly air sensitive and easily oxidized, it is taken as a prototype element in the present study. We hope that this study will attract interest in further study to find other elements, which form stable but air-insensitive ring structures. We also point out the technical difficulties in forming ring structures around a SWNT. However, recent advances in manipulating and relocating single atoms encourage us to explore the interesting features of these nanostructures.

\section{ACKNOWLEDGMENTS}

This work was partially supported by the NSF under Grant No. INT01-15021 and TUBÍTAK under Grant No. TBAG-U/13(101T010). Z.G. and S.C. acknowledge partial support from TÜBA/Gebip and TÜBA, respectively.
${ }^{1}$ N. Agrait, J. G. Rodrigo, and S. Vieira, Phys. Rev. B 47, 12345 (1993).

${ }^{2}$ For a current review of nanowires and carbon nanotubes, see $\mathrm{S}$. Ciraci, A. Buldum, and I. Batra, J. Phys.: Condens. Matter 13, 537 (2001).

${ }^{3}$ H. Ohnishi and Y. Kondo, K. Takayanagi, Nature (London) 395, 780 (1998); Y. Kondo and K. Takayanagi, Science 289, 606 (2000).

${ }^{4}$ A.I. Yanson, G. R. Bollinger, H.E. Brom, N. Agrait, and J.M. Ruitenbeek, Nature (London) 395, 783 (1998).

${ }^{5}$ H. Mehrez and S. Ciraci, Phys. Rev. B 56, 12632 (1997).

${ }^{6}$ O. Gülseren, F. Ercolessi, and E. Tosatti, Phys. Rev. Lett. 80, 3775 (1998).

${ }^{7}$ N. Agrait, G. Rubio, and S. Vieira, Phys. Rev. Lett. 74, 3995
(1995); 76, 2302 (1996).

${ }^{8}$ E. Tosatti, S. Prestipino, S. Kostlmeier, A. Dal Corso, and F. D. Di Tolla, Science 291, 288 (2001).

${ }^{9}$ Y. Zhang and H. Dai, Appl. Phys. Lett. 77, 3015 (2000).

${ }^{10}$ Y. Zhang, N. W. Franklin, R. J. Chen, and H. Dai, Chem. Phys. Lett. 331, 35 (2000).

${ }^{11}$ O. Gülseren, T. Yildirim, and S. Ciraci, Phys. Rev. Lett. 87, 116802 (2001).

${ }^{12}$ D. Srivastava, D. W. Brenner, J.D. Schall, K. D. Ausman, M. Yu, and R. S. Ruoff, J. Phys. Chem. B 103, 4330 (1999).

${ }^{13}$ O. Gülseren, T. Yildirim, and S. Ciraci, Phys. Rev. B 65, 155410 (2002); 65, 153405 (2002).

${ }^{14}$ A. Bezryadin, C. N. Lau, and M. Tinkham, Nature (London) 404, 971 (2000). 
${ }^{15}$ C. K. Yang, J. Zhao, and J. P. Lu, cond-mat/0202150 (unpublished).

${ }^{16}$ M. C. Payne, M. P. Teter, D. C. Allen, T. A. Arias, and J. D. Joannopoulos, Rev. Mod. Phys. 64, 1045 (1992).

${ }^{17}$ J. P. Perdew and Y. Wang, Phys. Rev. B 46, 6671 (1992).

${ }^{18}$ D. Vanderbilt, Phys. Rev. B 41, 7892 (1990).

${ }^{19}$ In Ref. 11 the binding energy of a single $\mathrm{Al}$ on the $(8,0)$ tube was calculated to be $\sim 1 \mathrm{eV}$. This is $0.7 \mathrm{eV}$ smaller than the present results. This is because in Ref. $11 E_{b}$ was calculated using a single unit cell incorporating significant $\mathrm{Al}-\mathrm{Al}$ interaction in $E_{T}[\mathrm{Al}]$ in Eq. (1).

${ }^{20}$ T. Yildirim, O. Gülseren and S. Ciraci, Phys. Rev. B 64, 075404 (2001).

${ }^{21}$ D. S. Portal, E. Artacho, J. Junquera, P. Ordejon, A. Garcia, and J.
M. Soler, Phys. Rev. Lett. 83, 3884 (1999).

${ }^{22}$ M. Okamoto and K. Takayanagi, Phys. Rev. B 60, 7808 (1999).

${ }^{23}$ F. D. Tolla, A. D. Corsa, J. A. Torres, and E. Tosatti, Surf. Sci. 454-456, 947 (2000).

${ }^{24}$ H. Häkkinen, R. N. Barnett, A. G. Scherbakov, and U. Landman, J. Phys. Chem. B 104, 9063 (2000).

${ }^{25}$ P. Sen, S. Ciraci, A. Buldum, and I. P. Batra, Phys. Rev. B 64, 195420 (2001).

${ }^{26}$ Y. Miyamoto, S. G. Louie, and M. Cohen, Phys. Rev. Lett. 76, 2121 (1996); Y. Miyamoto, Phys. Rev. B 54, 11149 (1996); Y. Miyamoto, A. Rubio, S. G. Louie, and M. L. Cohen, ibid. 60, 13885 (1999).

${ }^{27}$ J. R. Friedman, V. Patel, W. Chen, S. K. Tolpygo, and J. E. Lukens, Nature (London) 406, 43 (2000). 